\title{
The Biology of Nitric Oxide, Part 7
}

\author{
B Brüne \\ University of Erlangen-Nürnberg, Medical Department IV, Experimental Division, Loschgestrasse 8, 91054 Erlangen, Germany
}

The Biology of Nitric Oxide Part 7. Edited by S Moncada, LE Gustafsson, NP Wiklund, EA Higgs, Portland Press, London, UK: 2000, Pp. 234, £110, ISBN: 1855781425

This book is in the tradition of previously related titles (The Biology of Nitric Oxide, Parts 1-6) that cover cutting edge scientific contribution related to the chemistry, biology, and medicine of nitric oxide. Ten years after the initiating conference on a series of NO conventions, which was held in London in September 1989, this book now summarizes about 35 oral communications and approximately 300 poster presentations of the 6th International Meeting on the Biology of Nitric Oxide, which was organized in September 1999 in Stockholm, Sweden. With the editors, especially Professor Dr. S Moncada, we find an outstanding and remarkable active participant and scientific contributor to the field of nitric oxide, who overlooks the divers actions of $\mathrm{NO}$ and developmental changes from the very beginning.

NO is produced along with L-citrulline by the enzymatic oxidation of L-arginine. Since its discovery as a biologically active agent in the late 1980s, intensive investigations have demonstrated important roles of the rather small molecule (molecular weight of $30 \mathrm{D}$ ) in a range of physiological as well as pathophysiological processes. Constitutive forms of the NO-synthase (brain or endothelial type enzymes, known as nNOS versus eNOS) produce NO for short periods of seconds to minutes, whereas the inducible NOsynthase (iNOS) is expressed after cell activation and generates NO over an extended time of hours to days. In mammals, there is a rough correspondence between toxic and homeostatic functions of $\mathrm{NO}$ with the notion that reactive nitrogen intermediates (RNI) include not only $\mathrm{NO}$, the primary reaction product of NO-synthase isoenzymes, but also those species resulting from NO's rapid reduction, oxidation, or adduction in biological milieus. For signaling properties, various nitrogen oxides, S-nitrosothiols, and peroxynitrite (the reaction product of $\mathrm{NO}$ and superoxide) received considerable attention. NO controls a variety of complex biological processes, including blood pressure homeostasis, transmission of signals in the neuronal system, and carries out key roles in innate and specific immunity.

As stated in the preface of the book, S Moncada aims to the still expanding field of NO, touching areas, among others, of molecular biology, biochemistry, physiology, pathophysiology, inflammation, tumor biology, chemistry, clinical aspects, and apoptosis. The structure of the book roughly provides one page for each contribution which allows approximately 350 communications from various groups to be included in total. As a result, the aspects covered by this book are quite extensive and an up-to-date summary of the current status of the field is provided. This is excellent for insiders, looking for detailed information, new cross-links, or just being interested in the current research focus of individual groups. In this respect, most of the chapters are well organized, providing background information, technical comments, results, and a brief discussion. In most papers, figures or tables illustrate major findings and references provide information for more advanced reading on a particular topic. At the same time, the huge amount of data on the various topics of NOresearch may turn out as a disadvantage for the less experienced reader. It may appear difficult to discriminate between important and less thorough studies and one may get confused by contradictory findings on similar topics that sometime can be found next to each other. One should bear in mind that some excellent contributions appear as part of complex studies by groups with a long tradition in NO research, whereas other groups present descriptive and somehow unreflected observations at the very beginning of entering the NO field.

Based on the contributions that have been selected as oral communications we get some information on the topics that appeared most relevant to be presented to the conference audience. Assumingly, these contributions indicate the current scientific standing of the field and may point to future directions. Oral presentations can be grouped with respect to the following major themes:

(1) NO-synthase isoenzymes, structure, activation and regulation. Contributions covered (a) mapping of the active site of NO synthase by testing a range of structurally related L-arginine analogs that each preserve some but not all of the possible guanidinium region binding interactions; (b) the impact of 5-methyltetrahydrobiopterin in NO synthase activation; (c) the control of a putative calmodulin binding region to modulate eNOS activity; (d) formation of superoxide by neuronal NO synthase and its attenuation by tetrahydrobiopterin; and (e) regulation of neuronal NO synthase by calmodulin kinases that promote phosphorylation of nNOS at Ser847 in vitro and in vivo, thus altering the activity control of the enzyme.

(2) NO - donors and detection. Contributions covered (a) strategies for converting biomedically important substrates to NO-releasing forms; (b) visualization of NO 
formation by a NO-specific fluorescent probe; and (c) the use of laser spectroscopy for measurements of $\mathrm{NO}$, $\mathrm{NH}_{3}$, and other constituents in human breath.

(3) Mitochondrial complex I interactions and tissue respiration. Contributions covered (a) the reaction of $\mathrm{NO}$ with ubiquinone to prevent the activity of purified complex, I, thus attenuating oxidation of NADH; (b) inhibition of respiration at the level of complex I which is preceded by a decrease in the concentration of reduced glutathione; and (c) the impact of low level NO in regulating physiological mitochondrial respiration and the efficacy of high amounts of NO in blocking oxygen consumption in association with multiple organ failure and death.

(4) NO synthase, genes and transcriptional regulation. Contributions covered (a) genomic organization of the 5 -leader sequences and mRNA structure of the human nNOS gene with the finding of an unusual complex promoter multiplicity; (b) unique RNA diversity to mediate translational control efficacy in a tissue and differentiation-specific manner; and (c) dynamic regulation of endothelial NO synthase promoter activity by protein phosphatase $2 \mathrm{~A}$ complexed with casein kinase 2 to provide a fast, efficient on and off control of eNOS promoter activity.

(5) Targets of NO. Contributions covered (a) downstream effector systems of NO/cGMP signaling in the brain such as ion channels, phosphodiesterases, and protein kinases, types I and II; (b) S-nitrosylation of ornithine decarboxylase as a way to attenuate cell proliferation; (c) S-nitrosylation of a newly developed polyethylene glycol-conjugated hemoglobin, an artificial red blood cell substitute lacking side effects on vasoconstriction and arterial blood pressure; (d) reversible inhibition of nuclear vitamin $D_{3}$ and retinoid $X$ receptor heterodimer complex formation and dimerization by $\mathrm{NO}$ with implications of attenuated vitamin $D_{3}$ signaling under the influence of NO; (e) RNA viral mutations and evolution accelerated by NO-induced oxidative stress; (f) DNA microarray analysis to identify apoptotic genes under the positive or negative transcriptional control of $\mathrm{NO}$ in HL-60 cells; (g) denitrosylation of caspase-3 during Fas-induced apoptosis with the assumption of a reversible S-nitrosylation-denitrosylation equilibrium to regulate enzyme function; $(\mathrm{h})$ the role of glutamate/NO/ cGMP signaling pathways in the regulation of tyrosinase activity with concomitant melanin pigment formation in the ink gland of Sepia officinalis; (i) NO-evoked activation of $\mathrm{p} 21^{\text {Ras }}$ in a model of ischemic preconditioning that results in the expression of factors that protect neurons against subsequent lethal insults of ischemia; and (j) the ability of NO to inhibit tumor cellinduced platelet aggregation by attenuating the release of matrix metalloproteinase-2 from cancer cells via a cGMP-dependent mechanism.

(6) The use of NO synthase inhibitors. Contributions covered (a) the use of isolated organ systems to better predict in vivo selectivity for nNOS versus eNOS inhibitors compared to in vitro enzymatic assays; (b) the use of guanidinoethyldisulfide, a iNOS inhibitor and peroxynitrite scavenger, to prevent both autoimmune and oxidant-induced diabetes; (c) infusion of GW273629, a selective, short-acting iNOS inhibitor, in a mouse endotoxin shock model with the outcome of decreased mortality and increased survival time; (d) application of GW274150, a selective, long-acting iNOS inhibitor with therapeutic potential in post-operative ileus; and (e) the contribution of $\mathrm{NO}$ and related radical and oxidant species in the pathogenesis of inflammatory bowel disease in various animal models with the notion that mercaptoethylguanidine do not appear to consistently affect the course of the disease.

(7) NO in humans and disease states. Contributions covered (a) the finding that human monocytes and blood mononuclear cells from ill subjects (e.g. rheumatoid arthritis or patients with hepatitis) have increased iNOS expression and NO production; (b) evidence that iNOS expression occurred in macrophages located around capillaries in the human atherosclerotic plaque that may form the link between neovascularization and plaque destabilization; (c) supportive evidence for iNOS induction in multiple sclerosis with the assumption that NO production is an indicator of active disease, most likely involved in the pathogenesis of the disease state; (d) the expression of urease of Helicobacter pylori as a defence system against peroxynitrite via production of $\mathrm{CO}_{2} / \mathrm{HCO}_{3}{ }^{-}$, thus allowing to sustain infection of the bacterium in vivo; and (e) the findings that increased production of reactive oxygen and nitrogen species together with the decrease in the level of antioxidants, such as catalase, may render MRL-Ipr/lpr mice (mice that spontaneously develop manifestations of an autoimmune disease, some of which resemble human systemic lupus erythematosus and rheumatoid arthritis) more susceptible to oxidative damage and contribute to the pathogenesis of autoimmune disease; and (f) the conclusions that Bacillus Calmette-Guerin induced NOS activity and luminal NO concentrations in the urinary bladder. In turn, NO exert cytostatic effects on bladder cancer cells and induces apoptosis. NO synthesis may be an important mechanism in an anti-tumor therapy.

The selected themes for oral communications represent a fairly good cross-section of topics of current interest in the NO community. Papers from poster communications may roughly be grouped according to the major subjects as discussed. Among them, additional outstanding conceptual contributions are found that describe and add to the complexity of the role of $\mathrm{NO}$ in patho-physiology. In some cases, we learn more about the fine regulation of NO actions in established (test) systems, whereas in others we are confronted with additional players that modulate the signaling of NO.

The book is published quite timely, shows the complexity of transducing pathways that are affected by delivery or elimination of $\mathrm{NO}$ and provides a broad overview of key players. However, a deficit will and must remain since we are far from having a complete picture on the role of NO in acting as a direct effector molecule and/or as a modulator 
of others. In this respect the book provides a snapshot of current scientific knowledge and thinking. At this point, we anxiously wait for demanding answers on destructive and/ or beneficial roles of $\mathrm{NO}$ in defined experimental settings that will direct trials with NOS inhibitors, NOS-cDNAs, NO donors, or inhibitors that scavenge RNS. How all these pieces will eventually merge into a coherent picture is a question and task that will keep us busy in the future. Some answers will hopefully be given at the next NO conference, some new questions will appear, and some of our current knowledge will probably turn out to be too simplistic or incorrect. It is a scientific challenge that drives the NO field and joins together experts from various scientific disciplines. The biology of nitric oxide will remain a useful avenue for proceeding toward a more complete understanding of the NO production by human cells under physiological conditions and in various disease states. We may envision to substitute NO on demand or to eliminate or downregulate its production when produced in excess. Some answers are uncovered in this book. 